\title{
Digital divides across consumers of internet services in Spain using panel data 2007-2019. Narrowing or not?
}

\author{
Teodosio Pérez-Amaral, ICAE, UCM \\ Angel Valarezo, ICAE, UCM \\ Rafael López, ICAE, UCM \\ Teresa Garín-Muñoz, UNED
}

\begin{abstract}
Digital gaps have the potential to exacerbate the inequalities that exist in society. The main objective of this paper is to study the gaps that occur in the use of internet services by households in Spain during the period 2007-2019 and to become useful in the design of policies addressed to narrow specific digital divides. The data is a panel obtained from the ICT-H Survey on Households of the National Statistics Institute. This paper defines the gaps as the differences in the use of internet services across individual consumers. A selected group of twelve digital services are considered: e-commerce, e-selling, e-tourism, e-learning, e-health, ebanking, e-government, VoIP, chat, email, cloud services, and social networks. The second level digital gaps are analyzed in each service according to six relevant socio-demographic characteristics: gender, age, education, digital skills, population size, and income. A set of graphs show the evolution of the gaps. Gaps are narrowing in most dimensions and specific characteristics, but not in others such as age, education, and digital skills. The gaps reveal the evolution of digitization and in some cases of digital exclusion for specific groups. Specific knowledge about digital gaps is useful for policymakers, since closing the digital divide is an explicit policy goal in this country, as well as in other parts of Europe. Then, a dynamic panel data model was proposed and estimated using Arellano and Bond techniques. A dynamic/network effect was found, as well as other socio-demographic determinants. Finally, the paper contains conclusions, policy recommendations and an agenda for future research. The policy recommendations consist of digital education programs targeted at the most exposed groups such as the elderly, the less well-educated and people with lower digital skills.
\end{abstract}

Keywords: digital divide; digital gaps; internet services; individual panel data; dynamic panel data model.

JEL Classification: C30; L86; L96.

\section{ICAE Working Paper $n^{\circ} 2101$}

January, 2021 


\title{
Digital divides across consumers of internet services in Spain using panel
} data 2007-2019. Narrowing or not?

\author{
Teodosio Pérez-Amaral, ICAE, UCM \\ Angel Valarezo, ICAE, UCM \\ Rafael López, ICAE, UCM \\ Teresa Garín-Muñoz, UNED
}

- ICAE: Instituto Complutense de Análisis Económico, Spain

- UCM: Universidad Complutense de Madrid, Spain.

- UNED: National University at a Distance, Spain.

Acknowledgements: The collaboration of INE is gratefully acknowledged. Research funded by the Autonomous Community of Madrid, Spain (Project: H2019/HUM-5859, MIMA-CM, Mercados, Innovación y Medio Ambiente), and National R\&D Plan, Project: PID2019107161GB-C32, 2020-2023.

Keywords: digital divide; digital gaps; internet services; individual panel data; dynamic panel data model.

JEL Classification: C30; L86; L96. 


\begin{abstract}
Digital gaps have the potential to exacerbate the inequalities that exist in society. The main objective of this paper is to study the gaps that occur in the use of internet services by households in Spain during the period 2007-2019 and to become useful in the design of policies addressed to narrow specific digital divides. The data is a panel obtained from the ICT-H Survey on Households of the National Statistics Institute. This paper defines the gaps as the differences in the use of internet services across individual consumers. A selected group of twelve digital services are considered: e-commerce, e-selling, e-tourism, e-learning, e-health, e-banking, e-government, VoIP, chat, email, cloud services, and social networks. The second level digital gaps are analyzed in each service according to six relevant socio-demographic characteristics: gender, age, education, digital skills, population size, and income. A set of graphs show the evolution of the gaps. Gaps are narrowing in most dimensions and specific characteristics, but not in others such as age, education, and digital skills. The gaps reveal the evolution of digitization and in some cases of digital exclusion for specific groups. Specific knowledge about digital gaps is useful for policymakers, since closing the digital divide is an explicit policy goal in this country, as well as in other parts of Europe. Then, a dynamic panel data model was proposed and estimated using Arellano and Bond techniques. A dynamic/network effect was found, as well as other socio-demographic determinants. Finally, the paper contains conclusions, policy recommendations and an agenda for future research. The policy recommendations consist of digital education programs targeted at the most exposed groups such as the elderly, the less well-educated and people with lower digital skills.
\end{abstract}




\section{Introduction}

The internet is a powerful agent of change. Since the world-wide-web opened the internet in $1991^{1}$, its diffusion has been very rapid around the globe and across new services, and it has given rise to significant changes in everyday life. According to data provided by the International Telecommunications Union (ITU, 2019), almost half of the world's population was using the internet. However, there are relevant differences in access and use across geographic areas and groups of individuals which are called digital divides. The definition of digital divide has evolved in the last quarter of the last century. The National Telecommunications and Information Administration, NTIA (1995) focused its studies on infrastructure access. Later, literature on the topic moved on to consider access to technology and services. Currently, attention has shifted towards digital divides in new services provided through the internet, which are the focus of the current investigation.

The term "digital divide" refers to the gap between individuals, households, businesses and geographic areas at different socio-economic levels with regard both to their opportunities to access information and communication technologies (ICTs) and to their use of the internet for a wide variety of activities... The policy rationale for studying digital divides is the social benefits to be derived from the spillovers and positive externalities associated with diffusion and greater use of ICTs and related improvements to the skill base, OECD (2001, p. 5 and $6)$.

A large body of literature has emerged recently. Uni and multi-dimensional definitions and measurements of digital divides are some of the leading research questions (Srinuan \& Bohlin, 2011; Hargittai, 2001; Helsper and van Deursen, 2015; Zhao et al., 2014). The challenging concept of digital divide has been investigated from different disciplines such as psychology, economics, sociology, medicine, political science, and others.

Based on the overall assessment of the European Commission (2020), and DESI's (Digital Economy and Society Index) five dimensions ${ }^{2}$, Spain ranks $11^{\text {th }}$ out of $28 \mathrm{EU}$ countries, according to the 2020 edition of the index. The country performs well in almost all domains, except for Human Capital (rank $16^{\text {th }}$ ) on which it is below EU average. And, although Spain performed above the EU average on the overall use of the internet, Spanish internet users are not getting the full benefits of online services yet. The use of online banking, shopping online and selling online are below the EU average (European Commission, 2020).

The main objective of this paper is to study the gaps that occur in the use of internet services by households in Spain and to become useful in the design of policies addressed to narrow specific digital divides. This

\footnotetext{
${ }^{1}$ There is broad consensus in situating the birth of the World Wide Web in 1991 when Tim Berners Lee created it, combining three existing resources (HTML - Hypertext Markup Language, HTTP - Hypertext Transfer Protocol, URL - Uniform Resource Locator) with a new program called Web Browser.

2 1) Connectivity, 2) Human capital, 3) Use of internet services, 4) Integration of digital technology, and 5) Digital public services.
} 
would allow residents to enjoy the full benefits of the Information Society (OECD 2001, European Commission, 2020).

This paper focuses on three research questions: first, how large are the gaps in each service along with the different variables and levels; second, are the digital gaps for each service closing or widening along time; and, third, whether some gaps are disappearing over time.

This paper is a continuation to previous literature in the area, while it adopts a new focus and uses different techniques than previous research. Earlier research has concentrated on modelling the access (penetration) of each service, while here the focus is modelling the gaps in the use of digital services. A contribution of the paper is to measure specific digital gaps which are classified according to socio-demographic variables. The analysis identifies the gaps that are large and stable over time. Another contribution is to propose an equation that captures the dynamics of the gaps in Spain for the period 2008-2019.

The article's approach is the following:

1. Adopt a suitable measure of the digital gap. This measure compares the highest level of adoption (for each service) to each of the lower ones.

2. Consider the second-level digital divides in a variety of twelve leading services offered over the internet, like e-commerce, e-selling, e-tourism, e-learning, e-health, e-banking, e-government, voice-over IP, chat, email, cloud services, and social networks. We do not consider fixedbroadband and mobile- broadband, since they represent access (first-level digital divide) to the internet (Cerno and Pérez-Amaral, 2006). Here, we do not consider cross-border e-commerce since it is a subset of e-commerce and has been treated elsewhere (Valarezo et al., 2018).

3. In each of the services above, we've calculated the digital gaps according to six socio-demographic dimensions usually considered in the literature: gender, age, education, digital skills, population size where the individual lives (habitat), and income ${ }^{3}$. Each socio-demographic characteristic may have two, three, four, or more categories in the sample, depending on how the information was collected in the survey. These categories lead to one, two, three or more gaps for each service, which makes it necessary to be able to define and deal with several gaps for the same service.

An improvement over previous literature is that the present article focuses on individuals, adopting an economic perspective of the digital gaps. This article includes graphs that show the dynamics of the gaps. As well as an equation to characterize and synthesize the dynamics of the gaps.

The rest of the article goes as follows: Section 2 contains a literature review. Section 3 shows the data and methodology. Section 4 contains the empirical model and discussion of estimation results. Next, section 5 includes a discussion of the results. Finally, section 6 includes conclusions, policy recommendations, caveats, and further research.

\footnotetext{
${ }^{3}$ As suggested in Cerno and Pérez-Amaral (2006).
} 


\section{Literature review}

The literature on digital divide is vast across individuals, regions, countries, disciplines, and services. The summary below is limited in scope and follows a chronological order.

A report by the National Telecommunications and Information Administration, NTIA (1995) discusses the digital divides $^{4}$ (related to telephone, computer, and Internet use), and their evolution in the US using data for the previous 15 years. They define the digital divide ${ }^{5}$ and study its development along time.

Hacker and Mason (2003) argue that if researchers avoid the problem of ethical indifference concerning the analysis of data and pay more attention to the links of ethics with their findings related to digital divides, a better quality of research and policy considerations are likely. The paper claims that most analytical contributions on the digital divide tend to neglect ethical discussions, which "contaminates" studies and reports with ideological filtering.

Cava-Ferreruela and Alabau-Muñoz (2006) study digital divides, making extensive use of multivariate models and the associated diagnostic statistics.

Cerno and Pérez-Amaral (2006) study the demand for Internet access and services in Spain using microdata. They advance the types of dimensions that may generate divides, which are analogous to those in the current article.

Demoussis and Giannakopoulos (2006) deal with the determination and extent of Internet use as a facet of the digital divide in Europe. They use 2002-2003 individual cross-section data from a variety of European countries and focus on the decision to use or not use the internet, as well as how much to use it. This paper contributes insightful discussions on the right way to account for the availability of the internet and the relevant population groups concerned. A measure of digital skills is missing in the model. The estimates may have omitted variable biases.

Barzilai-Nahon (2006) criticizes policymakers who trust simple measures of the digital divides. The paper presents a conceptual definition of the digital divide and supports the use of comprehensive indices rather than monotopic ones. The article emphasizes that policymakers should consider the purpose of the tool, the level of observation, and the methodological approach to the data.

To understand a complex subject such as the digital divide, Vehovar et al. (2006) argue that standard methodological approaches are not sufficient, and measures must be considered in a multivariate setting.

\footnotetext{
4 "The gap between individuals, household, business and geographic areas at different socio-economic levels with regard to their opportunities to access information and communications technologies and to their use for a wide variety of activities" (OECD, 2001, pp. 8-9).

${ }^{5}$ A digital divide can be regarded as a situation in which the demand for access or use of a given digital service by a specific group is considered as insufficient (by policy makers or researchers), compared to that of other group of reference.
} 
Three methodological approaches are analyzed to measure digital divides: 1) Multivariate log-linear modelling allows addressing interactions among variables. 2) Compound indices summarize several variables into a single index. And 3) Time-distances methodology, to analyze the evolution of digital divides across time. The authors conclude that implementing these more sophisticated types of analyses often yields different conclusions vis-à-vis the usual bivariate comparisons.

Van Dijk (2006) reviews the literature on the digital divide for the period 2000-2005. The article deals with three questions. (1) Which inequalities does the digital gap concept refer to? (2) What is new about the gap of access and use of information and communications technologies as compared to other scarce goods and services in society? (3) Have different types of digital gaps arisen in the new information age? A general conclusion is that many cultural and family-related differences in our society at large show up again in computer and Internet use. The author points out that among the shortcomings of digital divide research is its lack of theory, conceptual definition, interdisciplinary approach, qualitative research and longitudinal research. “... it calls for extensive empirical research, longitudinal research and multivariate analyses” (p. 232).

Hilbert (2016) shows that access to digital communication is a moving target. He performs a cross country study using longitudinal data for 172 countries for the period 1986-2014. While the number of subscribers reaches saturation levels, the bandwidth divide continues to be a target unlikely ever to be solved. The bandwidth divide has first increased over time and then decreased during the period 2012-2014. In general, the bandwidth divide relates to the income divide, which is notoriously persistent. He points out the urgency to develop indicators to track the digital divide in terms of bandwidth.

Ono \& Zavodny (2007) analyze digital inequality in five countries using microdata. They focus on the use of personal computers at home. They find gaps that are more prevalent in Japan, South Korea and Singapore than in the US and Sweden. They also find that differences in IT usage along demographic and socio-economic dimensions reflect the extent of differences in other areas of the economy and society.

Hilbert (2011) studies digital divides and their definitions. The author analyses the adoption of a new service as a contagious process instead of an economic decision. This paper suggests that there are a variety of digital divides which cannot be added up in a significant way since it may be meaningless to synthesize into a single index. This paper poses several relevant questions but falls short of supplying useful answers.

Srinuan \& Bohlin (2011) offer a clarifying literature review in which they identify the main topics in the discussion, methodologies and conclusions of the literature. They point out the salient features of the digital divide and highlight the factors intervening in the multi-dimensional approach: income, skills, geography, education, family structure, age, occupation, marital status, and cost. They also deal with other factors that belong to the multi-perspective approach, among others: gender, language, culture, and race.

Zoroja (2011) studies the digital divide between West European and former communist countries. The 
article focuses on Internet usage, e-commerce, and e-government. The paper reports the average penetration rates for the three areas considered, aggregating the measures for the developed European countries and post-communist countries. The investigation finds that the group of developed countries shows a higher intensity of use than the other countries. The paper also discusses digital gaps and links them to socio-economic factors, especially education.

Bach et al. (2013) consider the digital divide across firms. They perform a literature review of selected papers that can be classified into three groups: ICT usage, then the adoption of Internet and broadband adoption, and then ICT usage for specific business purposes. Later they proceed to identify several factors that may condition the appearance of digital gaps among firms.

Salemink et al. (2017) analyze the relationship between ICT developments and rural development. This study is a review of 157 papers, focusing on the general conclusions to understand the potential impact of the coming NGA technology. The paper reports that access and inclusion are the two main aspects considered. The paper remarks that rural communities are less connected, and they are the most in need of improved connectivity to compensate for their remoteness.

Ruiz-Rodríguez et al. (2018) conduct a comparative analysis on the adoption and use of ICTs in firms and on the digital gap between them at a regional scale in Europe. The construction of a synthetic index of digital development for countries in the EU and Spanish regions allows them to claim that Spanish regions have a medium-high level of digital development in Europe. On the other hand, the digital divide of the regions is lower than that of other European countries.

Nishijima et al. (2017) show that the use of ICT involves the acquisition of skills. Thus, digital literacy is a complementary ability. The study adopts inequality indexes of internet access and mobile phone ownership to measure the use of ICT goods in Brazil. Their results indicate that the digital divide among individuals is decreasing quickly among Brazilians over time. However, there is room for policies related to mobile internet broadband access. Besides, digital illiteracy is one of the main determinants of digital divides in the country, especially among elderly individuals.

A paper by Szeles (2018) deals with the regional digital divide in the European Union. It uses panel data models at the levels of regions and countries, but no theoretical model is specified, which should have contributed to the choice of relevant predictors. Digital skills may be omitted, while education turns out to be insignificant in some models. On the other hand, the exogeneity of several variables is doubtful in several cases. The omission of relevant variables may also be an issue. The interpretation of the signs is problematic in some cases.

Pérez-Amaral et al. (2020) focus on modelling the penetration of e-commerce in Spain using individual panel data. They discuss the possible sources of differences in the adoption of e-commerce by different groups, like gender, age, education, digital skills, habitat, and income. These suggest dimensions along 
which to explore digital gaps. These factors may also influence other internet-related services.

There are some lessons and contributions in the literature that are worth mentioning. The first one is that the digital divide is multi-dimensional rather than unidimensional. Digital gaps may exist between the different levels (categories) within the variables of gender, age, studies, digital skills, habitat, income, geographical areas, and others.

Previous literature shows that the focus of the digital divide has been evolving. Part of the research considers several categories of digital divides. A first level related to access consisting of fixed telephony, dial-up internet, mobile telephony, broadband, computer, and bandwidth. A second level consists of the uses of services provided over the internet: e-commerce, e-tourism, e-learning, e-health, e-banking, egovernment, voice-over IP, chat, email, cloud services, and social networks, among others. A third level would be the proficiency of the different users and groups for using the above features efficiently and taking advantage of the opportunities opened by those services.

The approach of using bivariate comparisons between penetration rates is useful. Nevertheless, a multivariate approach may be appropriate for disentangling the effects of the variety of factors that determine the digital gaps.

Some of the contributions above use aggregate data, while others use cross-section, pool or panel data. Some others do not consider possible relevant dimensions, such as income and digital skills. Others deal with individual services (Pérez-Hernández and Sánchez-Mangas, 2011; Garín-Muñoz et al. 2019: Valarezo et al., 2020; and Pérez-Amaral et al. 2020). Due to these circumstances, policy recommendations may be somewhat limited. In the next sections, we present new results that fill some of the gaps in existing literature, while using a large data set, novel indicators, and techniques. The contribution of this article is to consider a variety of socio-demographic characteristics using several levels of each variable. This strategy is applied to a variety of services using panel data. The paper deals with individual panel data for Spain during 2007-2019; however, its approach and findings may generalize to other countries.

\section{The data and methodology}

This article uses annual data on individual dwellings from the survey on Equipment and Use of Information and Communications Technologies by Households from 2007 to 2019. This is the maximum span of data accessible at this point since the income variable is only available for 2008 to 2019. It was carried out by the Spanish National Statistical Institute (INE, 2020) following the guidelines of Eurostat. It is representative at both regional and national levels and includes an elevation factor. The primary data are available at INE (2020) and contain a variety of additional information and reports related to every aspect of the design, implementation, and statistics (except for the individual identifier). It is a rotating survey which includes 18,000-21,000 dwellings each year. The same residence is interviewed a maximum of four (consecutive) years, and around 30\% are replaced every year. Approximately $60 \%$ of the interviews are conducted by phone (CATI, computer-assisted telephone interview) and $40 \%$ in person (CAPI, computer- 
assisted personal interview). Following Eurostat's guidelines, similar surveys have been performed in the rest of the EU countries.

The statistics supplied by Eurostat and the Spanish National Statistics Institute (INE) refer only to the population between the ages of 15 and 74 for purposes of comparison across countries. However, the group of $75+$ is incorporated here due to its relevance and the availability of data. This decision includes an additional $17 \%$ to the sample and eliminates a potential source of sample selection bias.

The raw panel data underwent rigorous analysis and filtering to extract and homogenize the information about individuals uniquely identified throughout the observation period. The process goes as follows:

\section{Constructing a pool of data from the survey}

The information on dwellings for each year is publicly available in INE (2020). Each year includes a raw data file with the socio-demographic information of the members of each residence, a second data file with the responses to the survey, and an additional Excel file with the description of both raw data files.

Next, the variables are subject to a process of homogenization. The survey's questionnaire varies each year according to the evolving situation of ICT. It implies that some new variables emerge, and some old ones disappear. Besides, each year, the names of many variables change (in publicly available microdata, most variables are tagged after their ordinal situation in the questionnaire -which usually changes). To homogenize the variables, we developed a script in $\mathrm{R}$ that scrapped the description excel files for identifying, across years, the same variables with different names and different variables that were assigned the same name.

\section{Constructing the panel from the pool of data}

The pool data are obtained from the publicly available data. The next step converts a pool of dwellings into a panel data set of individuals. To do that it is necessary to incorporate the dwelling identifier supplied by the INE to the previous pool database.

However, we are interested in identifying individuals rather than dwellings (a dwelling participating in the survey several years may have different respondents across years). Socio-demographic information for each member of a residence, like gender and date of birth, was used as a filter. These criteria allow identifying whether the respondent from a specific dwelling was always the same individual or not.

A panel was obtained from the survey 2008-20196. It consists of 210,370 observations (corresponding to 97,859 different individuals) and more than 750 variables. The data used for all the calculations is obtained from the above panel. The panel effectively used for the graphs has 2,880 observations, including the 12

\footnotetext{
6 The year 2007 is used in the graphs, but not in the equation because the explanatory variable income is missing for 2007.
} 
services, a varying number of socio-demographic categories, and 12 years.

The individual socio-demographic and economic dimensions of digital gaps are shown in table 1 which shows the dimensions, the characteristics and categories of each variable considered in this article.

Table 1. Dimensions, variables, and categories related to digital gaps.

\begin{tabular}{|c|c|c|}
\hline Dimensions & $\begin{array}{l}\text { Characteristics/ } \\
\text { Variables }\end{array}$ & Categories or levels within each variable \\
\hline Sociodemographic & $\begin{array}{l}\text { Gender } \\
\text { Age } \\
\text { Population size } \\
\text { (Habitat) }\end{array}$ & $\begin{array}{l}2 \text { groups: } 1 \text { if male, } 0 \text { if female } \\
7 \text { groups: } 16-25,25-35,35-45,45-55,55-65 \text {, } \\
65-75,75 \text { or more. } \\
5 \text { groups: less than } 10,000 ; 10,000-20,000 \text {; } \\
\text { 20,000-50,000; 50,000-100,000; and } \\
100,000 \text { or more and province capitals. }\end{array}$ \\
\hline Individual skills & $\begin{array}{l}\text { Education } \\
\text { Digital Skills }\end{array}$ & $\begin{array}{l}4 \text { levels of study: None or Primary, } \\
\text { Secondary, Bachelor's Degree, and Master or } \\
\text { PhD. } \\
4 \text { levels: Low, Medium, High, and Very } \\
\text { High. }\end{array}$ \\
\hline Economic & $\begin{array}{l}\text { Income (monthly } \\
\text { net income) }\end{array}$ & $\begin{array}{l}4 \text { groups: Low, Medium, High, and Very } \\
\text { High. }\end{array}$ \\
\hline
\end{tabular}

Source: TICH survey of INE (2020).

Table 1 above contains, in the first column, the dimensions relevant for the gaps in this study: sociodemographic, individual skills, and economic characteristics. The second column contains the specific variables available in this data set for approximating each dimension. In the third column, the different levels or categories of each variable. The variables are coded as dummies to facilitate interpretation.

Most of the previous variables and dimensions are well-established in the literature, and this includes Digital Skills which are relevant according to the European Commission (2019) ${ }^{7}$, which are key elements to foster digital inclusion.

The digital skills variable used in this study is a synthetic index based on the former ${ }^{8}$ European Commission's Digital Skills Indicator, which accounts for the number and complexity of activities involving the adoption and use of digital equipment and Internet services (European Commission, 2019). The index covers four areas of competence: information and data literacy, communication and

\footnotetext{
${ }^{7}$ The other variables considered in this article are also relevant according to the European Commission, 2019).

${ }^{8}$ The later indicator includes information about ICT specialist employment and ICT graduates. This data is not available for the sample of this study.
} 
collaboration, problem-solving, and software skills for content manipulation.

The respondents reveal their capabilities by answering specific questions in each area. Information skills account for people who have copied or moved a file or folder, used internet storage space, searched for information online, searched for information about goods and services, and searched for information about health-related topics. Communication and collaboration areas are estimated by the declared use of email, social networks, telephone calls through the internet, and web sites for sharing own content. The problemsolving area of competence regards to transfer files between devices, installing software and apps, change the settings of the software, selling of goods or services through the internet, taking online courses, using online education material, and carrying out online banking activities. The software skills include using text processing software, spreadsheet software, software to edit audiovisual content, creating documents that integrate different files, writing a computer program using specialized programming language, and using a spreadsheet's advanced functions.

This paper uses four categories of digital skills: low, medium, high, and very high. These correspond to the quartiles of the percentage of tasks that an individual consumer declares to be able to perform. Table 2 contains the descriptive statistics for 2008 to 2019.

Table 2: Descriptive statistics 2008-2019

\begin{tabular}{|lccccc|}
\hline Variables & Obs. & Mean & Std. Dev. & Min. & Max. \\
Gap & 2440 & 44.155 & 32.481 & -39.184 & 100 \\
Gender Average & 2880 & 0.488 & 0.001 & 0.486 & 0.492 \\
Age Average & 2880 & 3.802 & 0.102 & 3.636 & 3.938 \\
Education Average & 2880 & 2.168 & 0.042 & 2.107 & 2.249 \\
Digital Skills Average & 2880 & 1.942 & 0.223 & 1.494 & 2.320 \\
Habitat Average & 2880 & 2.607 & 0.043 & 2.544 & 2.651 \\
Income Average & 2880 & 2.227 & 0.074 & 2.091 & 2.358 \\
\hline
\end{tabular}

Source: TICH survey of INE (2020).

Note: There are only 2440 observations on the Gap due to non-availability of data of some year for specific services, as noted in figures 1-6.

OECD (2001) deals specifically with digital divides. However, the report does not provide a precise definition of the digital divide or a method to compare the evolution of digital divides across time.

This literature mostly uses the digital gap and digital divide interchangeably. This paper uses "gap" as a generic name, while it reserves “digital divide” for differences that are considered harmful and might need policy intervention. They would be significant and persistent gaps relative to a sensitive variable.

Some definitions of penetrations and gaps are given below for clarification. 


\section{Penetrations and gaps}

The penetration of a service is the total number of users over the relevant population. Let there be

$\mathrm{h}=1, \ldots, \mathrm{H}$, levels of a specific variable that may be associated with a digital gap

(e.g., low, medium, high, and very high income).

$\mathrm{P}_{\mathrm{H}}$, the percentage penetration of a given service for the upper group of consumers $\mathrm{H}$.

$\mathrm{P}_{\mathrm{o}}$, the percentage penetration of a service for any other group of consumers $\mathrm{O}$.

\section{Definitions of gaps}

The difference between the penetration of the highest category $(\mathrm{H})$ and the penetration of another (lower) group (O) can be characterized using different measures:

1. $\mathrm{Gap}_{1}=\mathrm{P}_{\mathrm{H}}-\mathrm{P}_{\mathrm{O}}$, the difference of penetrations between the penetration of the highest category and the penetration of another (lower) category.

2. $\mathrm{Gap}_{2}=\mathrm{P}_{\mathrm{H}} / \mathrm{P}_{\mathrm{O}}$, the ratio of penetration of the highest category $\mathrm{H}$ and the penetration of a lower category $\mathrm{O}$.

3. $\mathrm{Gap}_{3}=\mathrm{P}_{\mathrm{O}} / \mathrm{P}_{\mathrm{H}}$, ratio of penetrations of the lower category $\mathrm{O}$ and the highest category $\mathrm{H}$.

4. $\mathrm{Gap}_{4}=\left(\mathrm{P}_{\mathrm{H}}-\mathrm{P}_{\mathrm{O}}\right) / \mathrm{P}_{\mathrm{O}}$, the difference of penetrations over the penetration of the lower category, which equals $\mathrm{Gap}_{2}-1$.

5. $\mathrm{Gap}_{5}=\left(\mathrm{P}_{\mathrm{H}}-\mathrm{P}_{\mathrm{O}}\right) / \mathrm{P}_{\mathrm{H}}$, the difference of penetrations over the penetration of the highest category, which equals $1-\mathrm{Gap}_{3}$.

Gap5 is an attractive measure of the digital gap. It is bounded between zero and one and admits a straightforward interpretation as the percentage difference in penetration for the category $\mathrm{O}$ of a given service relative to the penetration in the higher category H. Gap5 is a standard measure and will be used from now on. We drop the " 5 ” for convenience.

The data on gaps

This section contains the graphs of gaps of a variety of services: e-commerce, e-selling, e-tourism, elearning, e-health, e-banking, e-government, voice over IP, chat, email, cloud services, and social networks across different variables: gender, age, education, digital skills, habitat and income and the different levels of each variable.

The data on e-learning and e-health for 2012 and 2014 are missing in the corresponding surveys. The data on chat are missing between 2011 and 2018, both included. Data for cloud services are missing before 2014. The data above are missing because the related questions were not asked in the questionnaire in the mentioned years.

The following three research questions will be analyzed in the present section:

1. How large are the gaps in each service along with the different variables and levels?

2. Are the digital gaps for each service closing or widening along time? 
3. Are some gaps disappearing over time?

A set of graphs of the digital gaps over a selected group of services related to the internet are shown. The variables or reasons for possible gaps that we analyze here are the following: digital skills, income, gender, age, education, and population size (habitat) ${ }^{9}$.

\section{Digital skills}

Figure 1 below illustrates the gaps observed in different services concerning the differences in digital skills in four groups of consumers: those with low, medium, high, and very high digital skills.

1. Concerning the rest of the services, the group of low digital skills continues to have large gaps that show a slight tendency to decrease, except possibly in the VoIP, chat, email services and social networks ${ }^{10}$. These differences may be called skill divides or human capital divides.

2. In the rest of the categories (medium and high digital skills) the tendency towards smaller gaps is more clearly appreciated.

Those gaps showing a slight decrease coincide with services that may be more complex to use or less adapted for lower levels of digital skills. The concerns about digital skills are similar to those for Brasil, in Nishijima et al. (2017).

\section{Income}

Figure 2 shows the three gaps between the four categories considered in the income dimension. The general conclusions from the inspection of Figure 2 are:

1. The gaps are generally decreasing over time. The smallest gaps occur towards the end of the period (2019). Three services that show a more intense reduction of the gaps are VoIP, chat, and social networks.

2. The gaps in e-learning remain stable over time.

\section{Gender}

Figure 3 contains the gender gaps.

1. They are mostly unfavorable for females and substantial in some cases, like e-selling.

2. Nevertheless, they are often decreasing and become small towards the end of the period.

3. In some other instances, the gaps favor women, like in e-health, VoIP, and social networks.

\section{Age}

Figure 4 contains the graphs of the age gaps.

\footnotetext{
${ }^{9}$ The literature considers other dimensions of digital divides between individual or groups, such as religion, ethnicity, geography, and nationality that may be relevant in specific contexts.

${ }^{10}$ The increasing penetration and ease of use of services like WhatsApp, Facebook Messenger or Gmail, among others, may explain the exception.
} 
1. The uses of services, except possibly, e-government and email, continue to show significant agerelated digital divides.

2. The gaps for the groups $65-74$ and $75+$ are substantial and not decreasing rapidly. This behavior of the gaps may be a sign of an age divide.

3. For most of the services, the gaps for the groups 65-74 and 75+ are substantially different in favor of the first. This result justifies the inclusion of both groups usually studied as a single group of $65+$.

4. For the rest of the services, the tendency towards small and decreasing gaps is clear.

\section{Education}

Figure 5 shows the three gaps in the different services concerning the four levels of education considered here.

1. In most services, the category of none or primary studies (the upper line) tends to decrease slightly. This may be signaling an education gap.

2. The response of the two middle categories is more favorable, with generally decreasing sizes of the gaps and apparent convergence in most services.

\section{Population size (habitat)}

Figure 6 contains the gaps in different services, according to the population size where the individual lives $^{11}$.

1. The gaps in most of the 12 considered services are converging towards similar values within each service and closing downwards. However, areas with smaller populations tend to lag behind, possibly revealing a rural divide.

2. In particular, services such as e-learning and social networks still do not converge; they remain between 5\% and 33\% for e-learning.

3. The presence of a strong habitat divide is not self-evident in this sample possibly because the data are not granular enough for small and very small populations.

Population and geographic gaps have been analyzed by Salemink et al. (2017), Szeles (2018), Zoroja (2011), Ruiz-Rodríguez et al. (2018), and Ono and Zavodny (2007). Their analysis is mostly performed at the regional and national levels while the analysis in the present article is performed at the individual level.

\footnotetext{
${ }^{11}$ The data of INE refer to the size of the population of the town and not necessarily the municipality.
} 


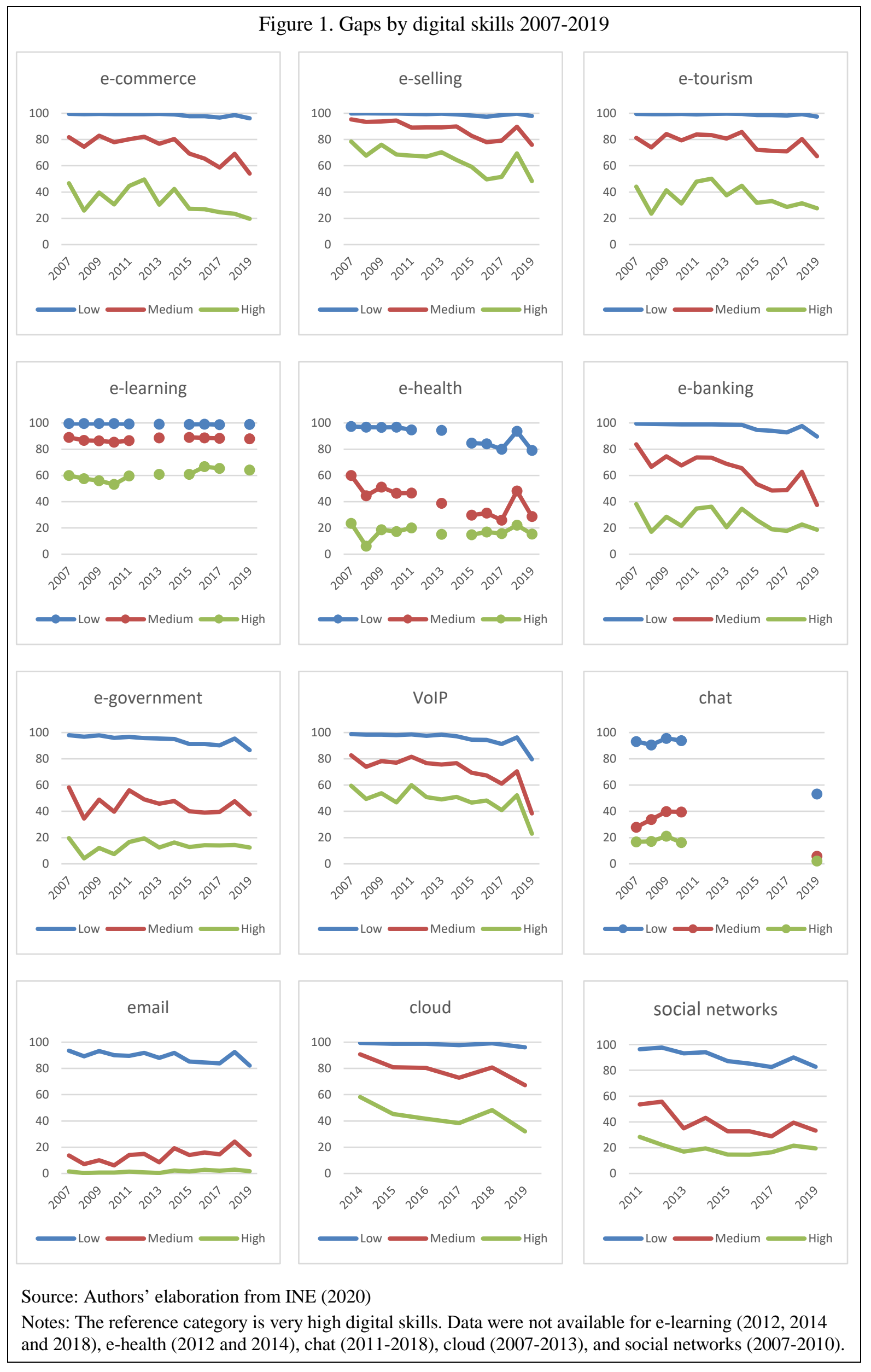




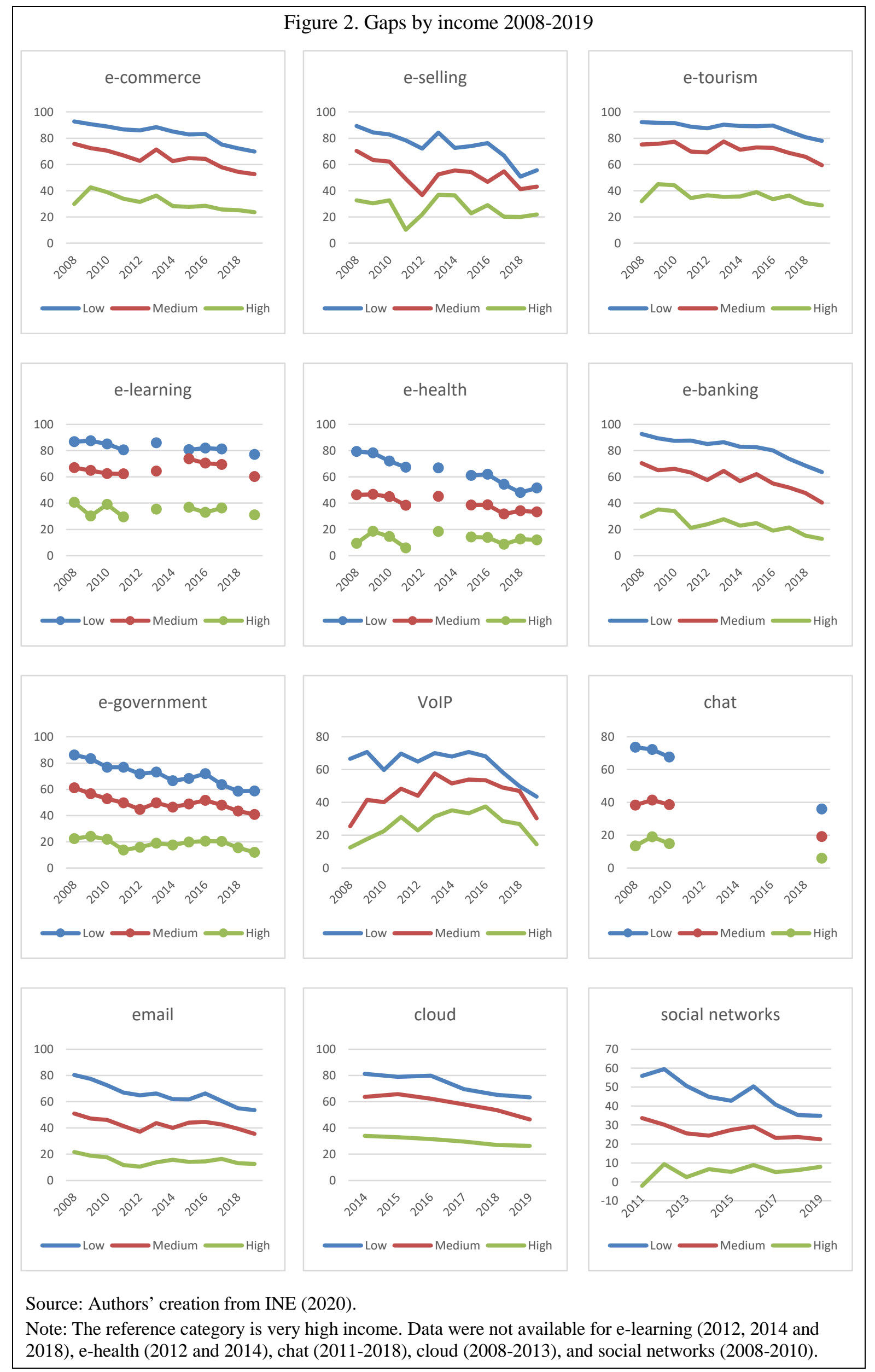


Figure 3. Gaps by gender 2007-2019
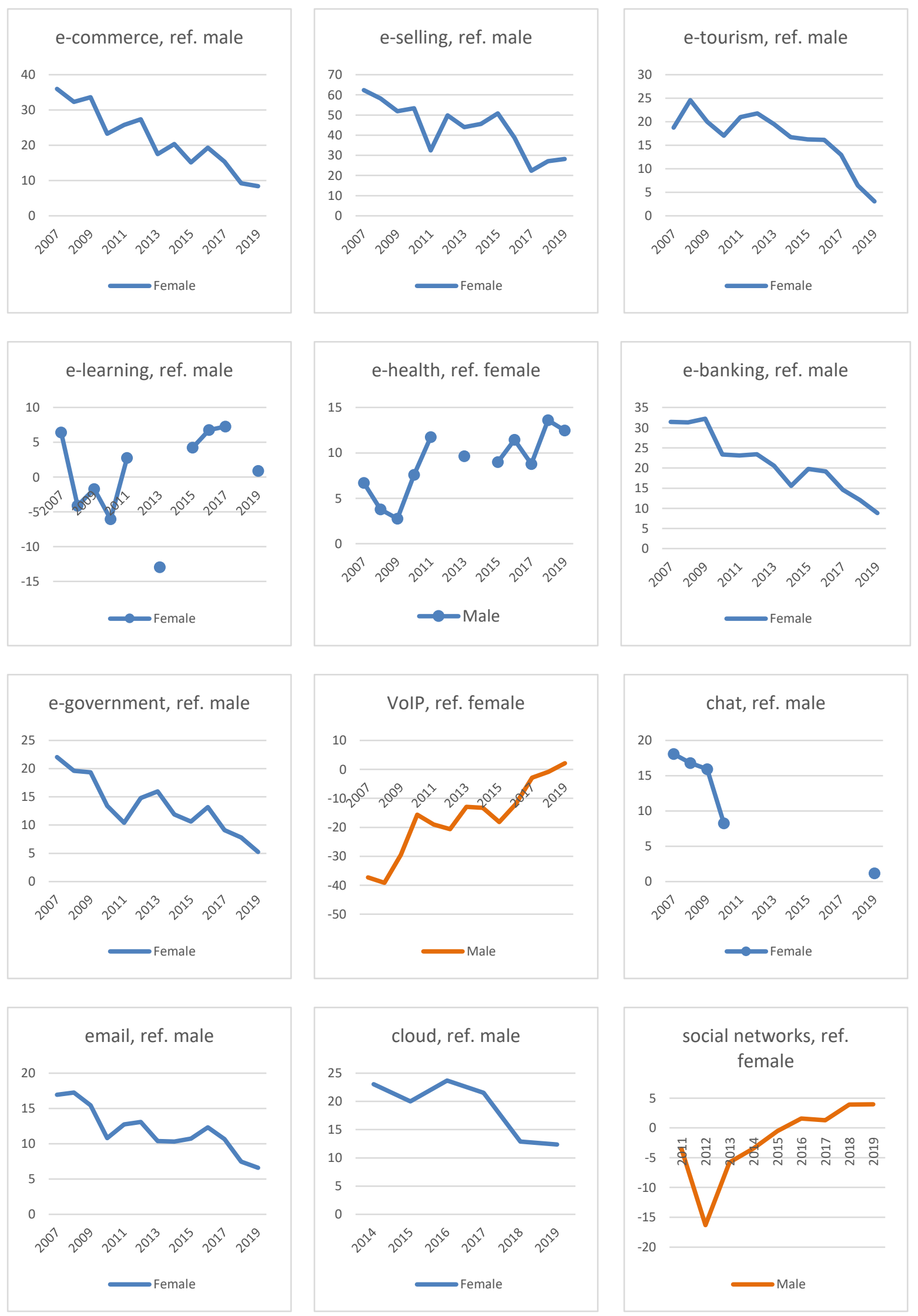

Source: Authors' creation from INE (2020).

N otes: The reference category is male, except for e-health, VoIP and social networks. Data were not available for e-learning (2012, 2014 and 2018), e-health (2012 and 2014), chat (2011-2018), cloud (20072013), and social networks (2007-2010). 


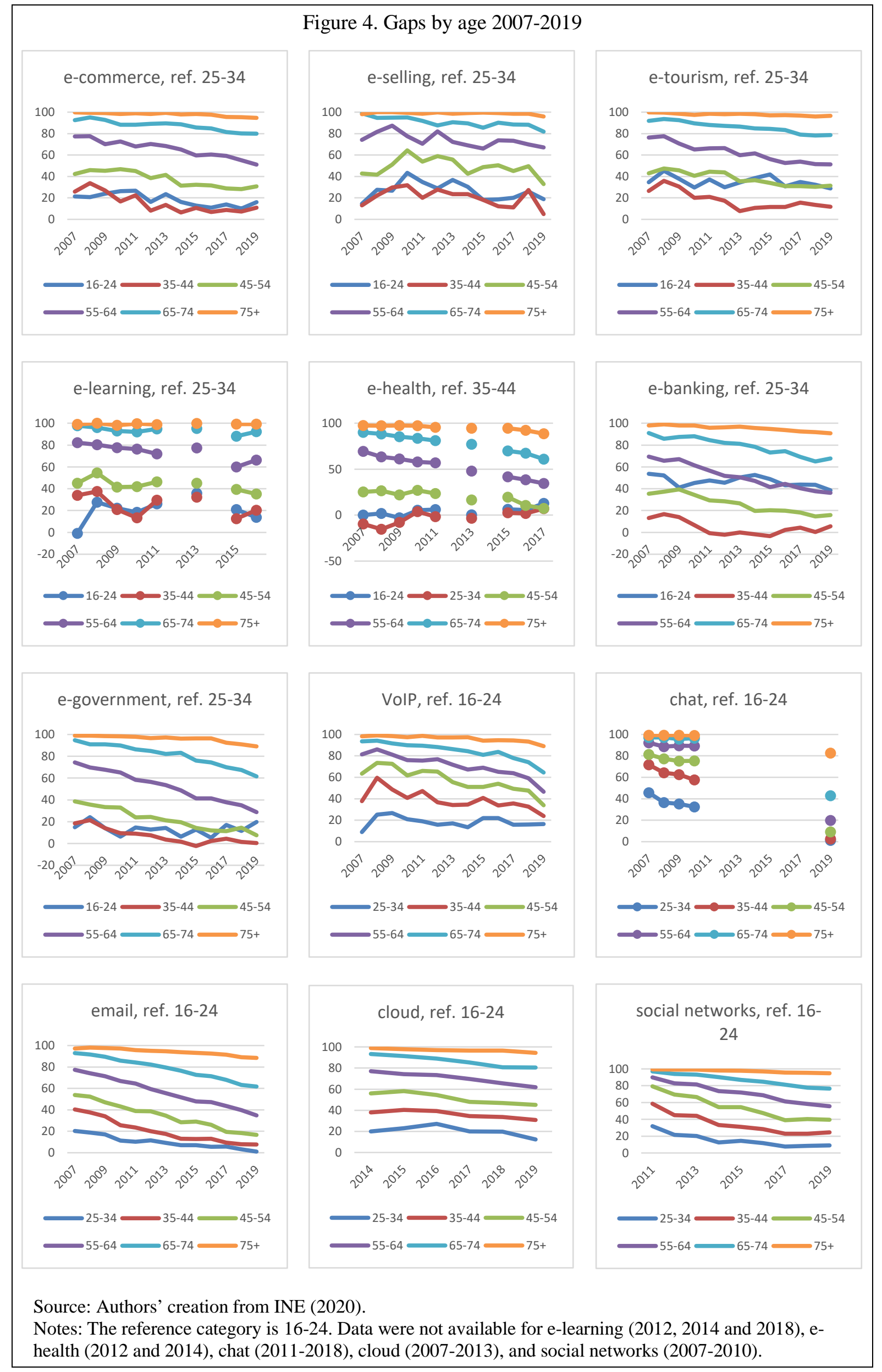




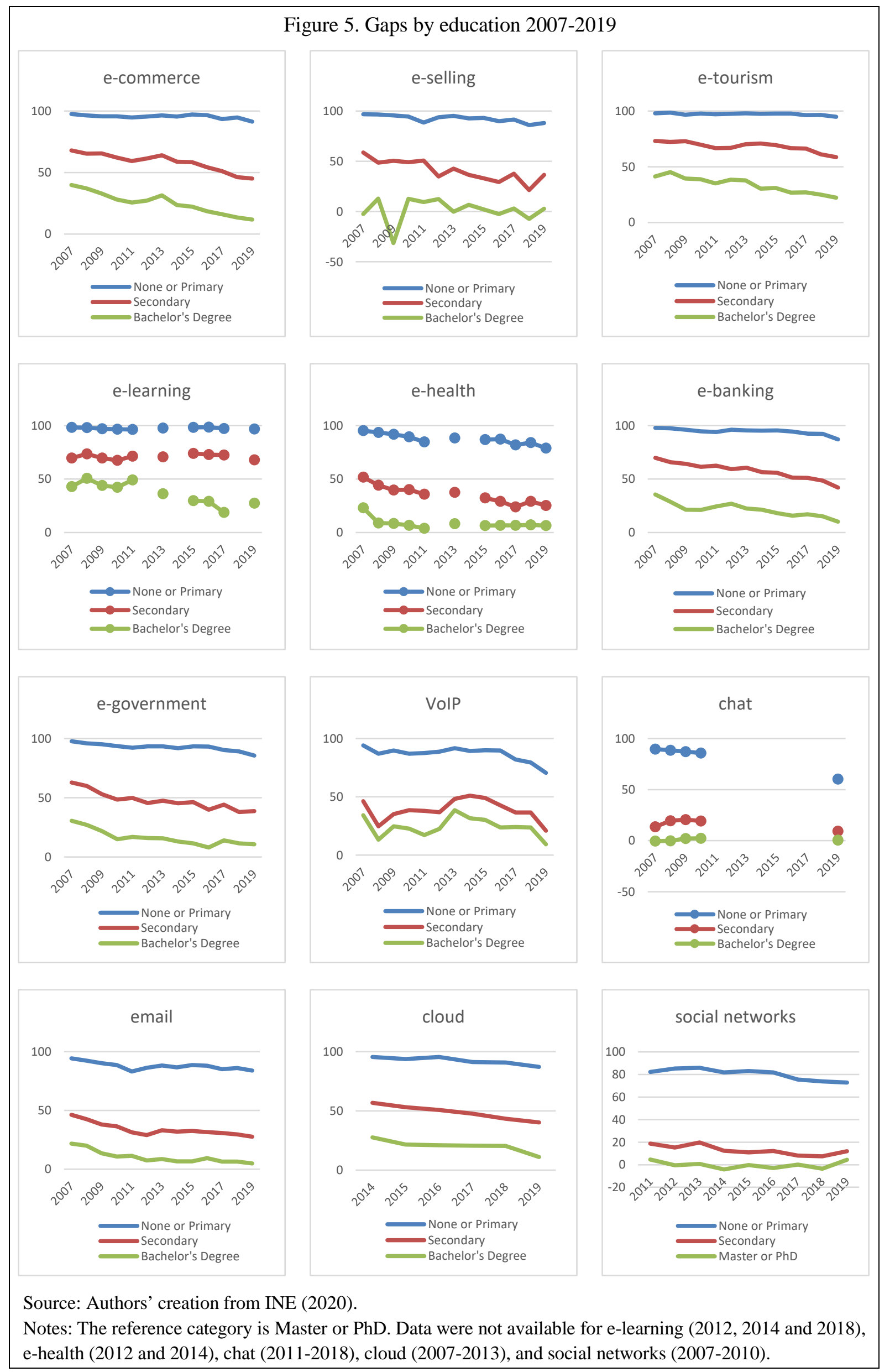


Figure 6. Gaps by habitat 2007-2019

e-commerce, ref. $100,000+$ and CCPP

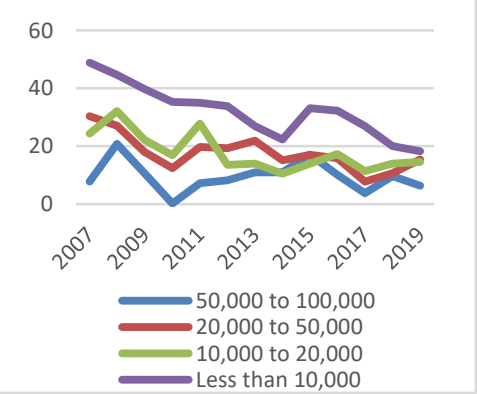

e-learning, ref. 100,000+ and CCPP

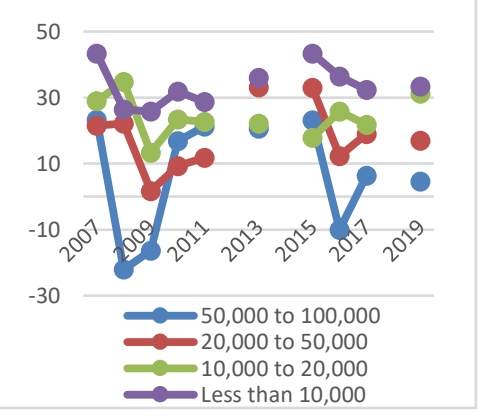

e-government, ref. $100,000+$ and CCPP

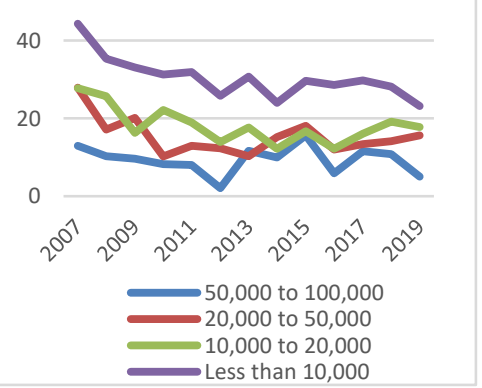

email, ref. 50,000 to 100,000

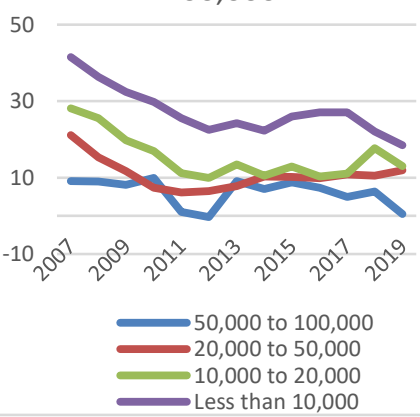

e-selling, ref. 100,000+ and CCPP



e-health, ref. 100,000+ and CCPP

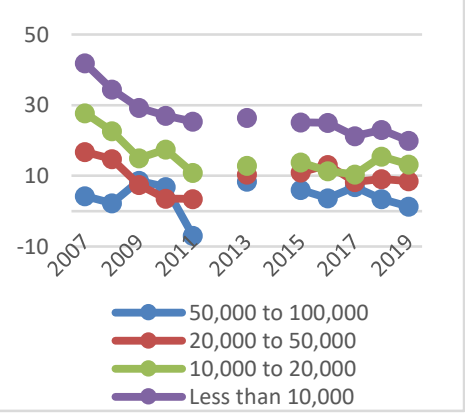

VolP, ref. 50,000 to 100,000

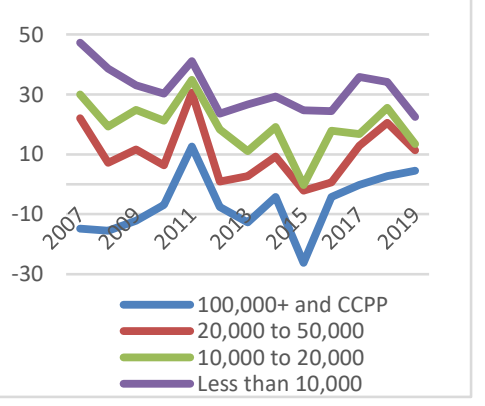

cloud, ref. 50,000 to 100,000

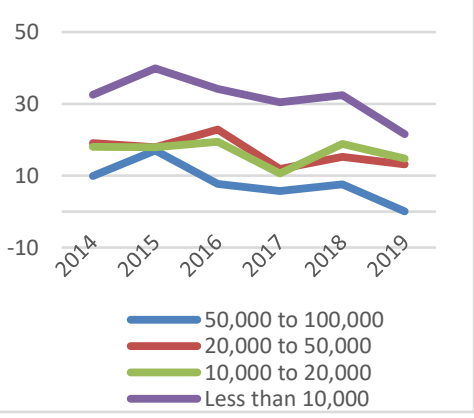

e-tourism, ref. 100,000+ and CCPP

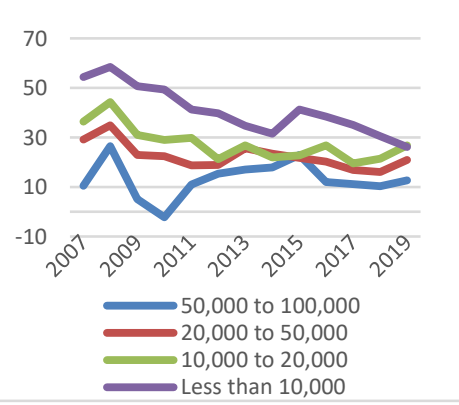

e-banking, ref. 100,000+ and CCPP

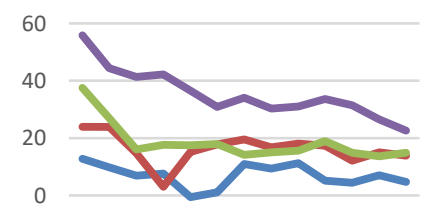

$-200^{0 \hat{0}} \nu^{0^{9}} \nu^{0^{2}} \nu^{0^{3}} \nu^{0^{3}} \nu^{0^{\hat{y}}} \nu^{0^{9}}$

50,000 to 100,000

20,000 to 50,000 10,000 to 20,000 Less than 10,000

chat, ref. 50,000 to 100,000

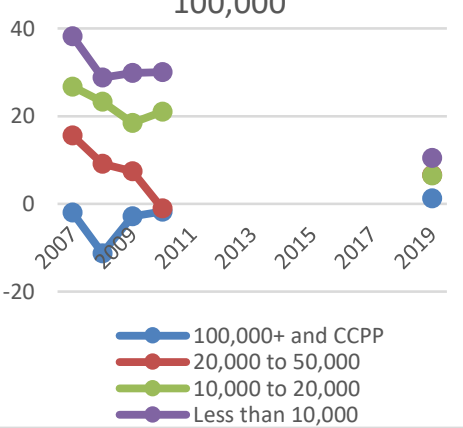

social networks, ref. 50,000 to 100,000

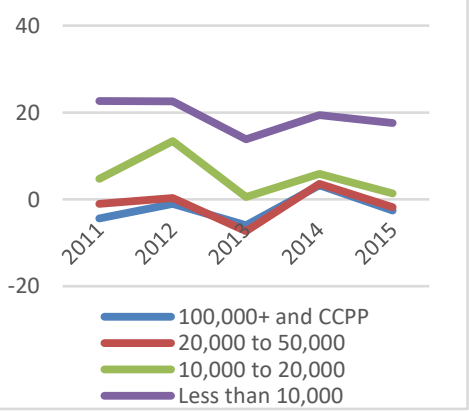

Source: Authors' creation from INE (2020).

Notes: Reference categories are indicated for each service. Data were not available for e-learning (2012, 2014 and 2018), e-health (2012 and 2014), chat (2011-2018), cloud (2007-2013), and social networks (2007-2010). 


\section{The empirical model and discussion of estimation results.}

The previous results suggest whether it would be possible to forecast the evolution of the gaps. Why are some gaps narrowing while some others persist over time? The following aggregate model synthesizes the joint evolution of the gaps in the 12 services across all variables and categories. The model considered here follows the spirit of Garín-Muñoz et al. (2019) and Pérez-Amaral et al. (2020), which are centered on the explanation of the penetrations. The gaps here are the differences in penetrations for different sociodemographic categories.

The gap for a given service concerning a variable and category at a given point in time is ${ }^{12}$

Gap $=\left(\mathrm{P}_{\mathrm{H}}-\mathrm{P}_{\mathrm{O}}\right) / \mathrm{P}_{\mathrm{H}}=1-\left(\mathrm{P}_{\mathrm{O}} / \mathrm{P}_{\mathrm{H}}\right)^{13}$

The specification of the model of the ratio $\left(\mathrm{P}_{\mathrm{O}} / \mathrm{P}_{\mathrm{H}}\right)$, can incorporate the same variables as those for modelling both $\mathrm{P}_{\mathrm{O}}$ and $\mathrm{P}_{\mathrm{H}}$. These explanatory variables are the levels of each of those variables in a model for penetration: gender, age, education, digital skills, habitat, income, employment, nationality, number of household members, plus a lagged dependent variable.

Since the model is specified at an aggregate level, the explanatory variables will be the sample averages calculated across all the individuals of the sample. The model is a panel of gaps across different services, variables, and levels within each variable. While the levels and dynamics of the gaps are unconditionally heterogeneous (different constants), the effects of each explanatory variable are assumed to be similar across all gaps.

The model used is dynamic, since it models gaps which are differences between processes that are dynamic per se. The gaps show a clear persistence along time, as seen in figures 1-6, suggesting a time-dependence that could be captured by an autoregressive (AR) process.

In the dynamic model, a lagged dependent variable, which is highly significant, captures network effects and dynamics. In any case, static models were also estimated, but they were less satisfactory in terms of goodness of fit.

The equation that we estimate in this section is as follows:

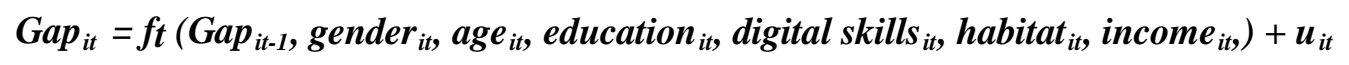

In table 3 we present the estimation of a linear version of equation 2 using averages of each explanatory variables as regressors.

${ }^{12} \mathrm{P}_{\mathrm{H}}=$ the penetration of the highest category and $\mathrm{P}_{\mathrm{O}}=$ the penetration of another (lower) category.

${ }^{13}$ This is Gap5 of the previous section. 
Table 3: Dynamic model of gaps. Panel data 2008-2019

\begin{tabular}{|c|c|c|c|}
\hline \multirow[t]{2}{*}{ Dependent variable: Gap } & \multicolumn{2}{|c|}{$\begin{array}{l}\text { Dynamic model } \\
\text { (in differences) }\end{array}$} & \multirow[t]{2}{*}{ p-value } \\
\hline & Coefficient & $\mathrm{z}$ & \\
\hline $1^{\text {st }}$ Lag of Gap & $0.26 * * *$ & 5.52 & 0.000 \\
\hline Gender Average & $-3.40 * * *$ & -3.47 & 0.001 \\
\hline Age Average & $-9.69 * * *$ & -4.13 & 0.000 \\
\hline Education Average & -1.87 & -0.30 & 0.764 \\
\hline Digital Skills Average & 0.34 & 0.28 & 0.781 \\
\hline Habitat Average & -1.54 & -1.45 & 0.147 \\
\hline Income Average & $-7.50 *$ & -1.91 & 0.057 \\
\hline Number of observations & & 1,801 & \\
\hline Number of groups & & 240 & \\
\hline Number of instruments & & 62 & \\
\hline Wald $\chi^{2}(7)$ [Prob. $\left.>\chi^{2}=0.000\right]$ & & 906.23 & \\
\hline \multicolumn{4}{|c|}{$\begin{array}{l}\text { Notes: }(1) * * * \mathrm{p}<0.01,{ }^{* *} \mathrm{p}<0.05,{ }^{*} \mathrm{p}<0.1 \text {. } \\
\text { (2) z-statistics are asymptotic approximations to the t's. They are asymptotically } \mathrm{N}(0,1) \\
\text { under the null. } \\
\text { (3) Estimated by GMM, using the Arellano and Bond procedure. } \\
\text { (4) The sample refers to individuals over } 15 \text { years of age. } \\
\text { Estimations performed in Stata } 16 \text {. }\end{array}$} \\
\hline
\end{tabular}

Each regressor is the average of the variable across the sample of INE in each year. The operating assumption is that the dynamics of the gaps are similar across services.

This model for the gap incorporates the first lag of the gap and the explanatory variables. The equation was estimated by the Generalized Method of Moments of Arellano and Bond, which used 62 instruments. The coefficients are jointly very significant, with a Wald test of 906.23, and a p-value of 0.0000 . This equation may be used for forecasting, so the coefficients measure in-sample correlations, rather than causation ${ }^{14}$. The estimate of the coefficient of the lagged dependent variable is $0.26(5.52)^{15}$. This estimate is capturing the dynamics, inertia effects, and network effects that are present in the evolution of the gaps. The decrease in a gap tends to be followed (ceteris paribus) by another decrease of .26 of its size.

\footnotetext{
${ }^{14}$ This approach is related to the spirit of Cava-Ferreruela and Alabau-Muñoz (2006), except here a variety of services and divides are used.

${ }^{15} \mathrm{z}$-statistics in parenthesis. The z-statistics are the asymptotic version of the t-statistics.
} 
The coefficient of gender average, -3.40 (-3.47) captures the effect of gender. As there is an increase in the average proportion of women, this is associated with smaller gaps, and it is consistent with the previous results regarding the decrease of most of the gaps for women.

The coefficient estimated for age average -9.69 (-4.13) captures the partial effect of the average age. The higher average age is associated with smaller gaps. This effect may be non-monotonic along with the different age groups.

The coefficient of habitat average, $-1.54(-1.45)$ is statistically insignificant. That is the reason why we do not interpret this coefficient.

The estimate -7.50 (-1.91) measures the association between an increase in average income across time and a decrease in the gap. The higher the increase in average income, is associated with smaller gaps. This is marginally significant, so this interpretation is only preliminary.

The coefficients of education and digital skills are not significant individually even though these variables are relevant in models of individual adoption of digital services (Garín-Muñoz et al., 2019; and PérezAmaral et al., 2020). This result may point out that education and digital skills have little variation along time. It may also suggest a disaggregation by services. Several of the effects are individually significant while others (habitat, education, and digital skills) are insignificant and appear not to be useful in this equation.

This dynamic model is going to be useful for short-run forecasting of gaps. The methodology for that exercise would consist in performing short-run forecasts for 2020 of the gaps using the information up to 2019 and then compare it with the actual data in 2020 when it becomes available. Using the short-run forecasts would allow detecting changes in gaps that may be due to different policies or special events such as pandemics. A comparison of the forecasts using information up to 2019 with the actual data on 2020 and 2021 is deferred for further research when the new data becomes available.

\section{Conclusions}

This article focuses on the digital gaps in Spain for the period 2007-2019. A panel of 97,859 individual consumers is used to analyze a variety of digital gaps in several internet-related services. The usual procedure in the literature is to compute one gap per service and variable. A novelty of this study is that all available gaps are considered in each service and variable. For instance, since there are four categories of income, a total of three gaps are computed for a given service. This perspective offers policymakers a richer view of the existing digital gaps and its evolution and allows for policy measures that are better targeted than more general policies. 


\section{Main conclusions}

Most gaps are narrowing as shown Figures 1-6, and in several cases, they become small. In the case of gender, the gaps, although large at the beginning, end up small or reversing in signs in some cases, likewise for VoIP, chat, and social networks. In the variable age, most gaps in several services tend to decrease. However, some gaps concerning older groups persist throughout the sample. The differences for the categories of 65-74 and 75 or more are not decreasing over time, and remain very high, around 60-100\%.

The gaps concerning education tend to decrease for most services and periods. However, a digital divide which remains high and stable over the years, around $80-100 \%$, can be identified, corresponding to a low level of education. Regarding digital skills, some decrease occurs for most categories and services; however, there exist digital divides between low digital skills and the rest of the groups that amount to $80-100 \%$ for all services.

Concerning habitat, the gaps are small and decreasing, nearing convergence in many services. This convergence occurs despite a more limited deployment of internet-related infrastructure in rural areas. Population and geographic gaps have been analyzed by Salemink et al. (2017), Szeles (2018), Zoroja (2011), Ruiz-Rodríguez et al. (2018), and Ono and Zavodny (2007). The gaps concerning income are decreasing, and most are becoming small. Still, there is an income divide for people with respect to lower income, which is around $60 \%$ in many services.

The gaps showing more stable evolutions coincide with services that may be more complex to use, especially for low digital skills or older consumers. These results are similar to those of Brazil (Nishijima et al. 2017).

The main pockets of digital exclusion concentrate on people with higher age, low education and low digital skills. After an appropriate cost-benefit analysis, it is necessary to make decisions on how to advance in the policy goal of “not leaving anyone behind”.

The second part of this article presents a forecasting equation designed for a short- term prediction of the evolution of the gaps. It is a model for panel data, formulated in differences, that describes the dynamics of the gaps and is estimated by GMM. This equation incorporates a dynamic AR term that captures the network and learning effects which are relevant for forecasting the gaps. This model reveals that the variables that correlate with a decrease of the gaps along time are gender, age, and income, as well as the dynamic term.

\section{Policy recommendations}


The results of this article suggest several policy recommendations. They consist of demand-side programs aimed at first improving the digital skills of specific socio-demographic groups which may be at risk of digital exclusion. With respect to age, it would be possible to introduce programs targeted at senior citizens, aiming to teach the basics of chat, e-commerce, news, and entertainment use that would significantly increase their quality of life. Remote support by phone may be very useful for these purposes. Similar digital education programs could be established with respect to persons with lower education.

These programs may require the prioritization of the recipients, given the existing financial constraints. A criterion for eligibility could be the prospective employability of the trainees. In the cases of education and digital skills, the programs may be more targeted on basic professional skills, in contrast with the program for senior citizens which would be more centered in private consumption and entertainment. Some current or previous programs in Spain with this flavor are COMPETIC (aimed at training adults in digital skills), CODEMADRID (a teacher training program), CODIX (a digital inclusion program open for the general public), IKANOS (to promote the dissemination of the European Digital Competencies Framework), TALENTUM SCHOOLS (to promote the digital vocation of students and boost digital talent among young people), and 42 MADRID (an open program, mostly for developing advanced digital skills in young people) (Garín-Muñoz et al. 2019; and Valarezo, et al. 2020).

\section{Caveats}

These conclusions are subject to caveats. The data set is large, and of high quality, but it is declarative, which may present difficulties of recollection. A specific survey would be desirable, but hard to finance. A disaggregation by different groups of services could possibly enhance the accuracy of the results and suggest more specific policy applications.

\section{Further research}

Some topics for further research are the dynamics of the gaps disaggregated by groups of services e.g., those that converge and those that do not, or b2b and b2c. Access services, such as fixed broadband and mobile broadband, are also going to be considered. Another line of research is to forecast out-of-sample and calculate the prediction errors when the actual data are available in 2021. Explaining the forecast errors would be useful to identify the effects of specific policy programs or unusual events. The comparison with an alternative Big Data non-parametric method for generating out-of-sample forecasts, in particular RETINA (Pérez-Amaral et al., 2003), is also a feasible task. 


\section{References}

Bach, M. P., Zoroja, J., \& Vukšić, V. B. (2013). Determinants of firms’ Digital Divide: A Review of Recent Research. Procedia Technology, 9, 120-128. https://doi.org/10.1016/j.protcy.2013.12.013

Barzilai-Nahon, K. (2006). Gaps and Bits: Conceptualising Measurements for Digital Divide/s. The Information Society, 22(5), 269-278. https://doi.org/10.1080/01972240600903953

Cava-Ferreruela, I., \& Alabau-Muñoz, A. (2006). Broadband policy assessment: A crossnational empirical analysis. Telecommunications Policy, 30(8), 445-

463. https://doi.org/10.1016/j.telpol.2005.12.002

Cerno, L., \& Pérez-Amaral, T. (2006). Demand for Internet Access and Use in Spain. In B. Preissl \& J. Müller (Eds.), Governance of Communication Networks: Connecting Societies and Markets with I.T. (pp. 333-353). Physica-Verlag HD. https://doi.org/10.1007/3-7908-1746-5_18

Demoussis, M., \& Giannakopoulos, N. (2006). Facets of the digital divide in Europe: Determination and extent of internet use. Economics of Innovation and New Technology, 15(3), 235-246. https://doi.org/10.1080/10438590500216016

European Commission. (2019). Human Capital and Digital Skills [Text]. Digital Single Market- European Commission. https://ec.europa.eu/digital-single-market/en/human-capital

European Commission. (2020). The Digital Economy and Society Index (DESI). Shaping Europe’s Digital Future - European Commission. https://ec.europa.eu/digital-single-market/en/desi

Garín-Muñoz, T., López, R., Pérez-Amaral, T., Herguera, I., \& Valarezo, Á. (2019). Models for individual adoption of eCommerce, eBanking and eGovernment in Spain. Telecommunications Policy, 43(1), 100-111. https://doi.org/10.1016/j.telpol.2018.01.002

Hacker, K. L., \& Mason, S. M. (2003). Ethical gaps in studies of the digital divide. Ethics and Information Technology, 5(2), 99-115. https://doi.org/10.1023/A:1024968602974

Hargittai, E. (2001). Second-Level Digital Divide: Mapping Differences in People’s Online Skills. ArXiv:Cs/0109068. http://arxiv.org/abs/cs/0109068

Helsper, E. J., \& van Deursen, A. J. A. M. (2015). The Third-Level Digital Divide: Who Benefits Most from Being Online? In Communication and Information Technologies Annual (Vol. 10, pp. 29-52). Emerald Group Publishing Limited. https://doi.org/10.1108/S2050-206020150000010002

Hilbert, M. (2011). The end justifies the definition: The manifold outlooks on the digital divide and their practical usefulness for policy-making. Telecommunications Policy, 35(8), 715-736.

https://doi.org/10.1016/j.telpol.2011.06.012

Hilbert, M. (2016). The bad news is that the digital access divide is here to stay: Domestically installed bandwidths among 172 countries for 1986-2014. Telecommunications Policy, 40(6), 567-581. https://doi.org/10.1016/j.telpol.2016.01.006

INE. Instituto Nacional de Estadística. National Statistics Institute. Spanish Statistical Office. (2020). Encuesta sobre equipamiento y uso de tecnologías de información y comunicación en los hogares. INE. https://www.ine.es/dyngs/INEbase/es/operacion.htm?c=Estadistica_C\&cid=1254736176741\&menu=ulti Datos\&idp $=1254735576692$ 
International Telecommunication Union (ITU). (2019). Measuring digital development: Facts and figures 2019. https://www.itu.int/en/ITU-D/Statistics/Pages/facts/default.aspx

National Telecommunications and Information Administration, NTIA. (1995). FALLING THROUGH THE NET: A Survey of the "Have Nots" "in Rural and Urban America.

https://www.ntia.doc.gov/ntiahome/fallingthru.html

Nishijima, M., Ivanauskas, T. M., \& Sarti, F. M. (2017). Evolution and determinants of digital divide in Brazil (2005-2013). Telecommunications Policy, 41(1), 12-24.

https://doi.org/10.1016/j.telpol.2016.10.004

OECD. (2001). Understanding the Digital Divide (OECD Digital Economy Paper No. 49). OECD Publishing. https://doi.org/10.1787/236405667766

Ono, H., \& Zavodny, M. (2007). Digital inequality: A five country comparison using microdata. Social Science Research, 36(3), 1135-1155. https://doi.org/10.1016/j.ssresearch.2006.09.001

Pérez-Hernández, J., \& Sánchez-Mangas, R. (2011). To have or not to have Internet at home: Implications for online shopping. Information Economics and Policy, 23(3), 213-226.

https://doi.org/10.1016/j.infoecopol.2011.03.003

Pérez-Amaral, T., Gallo, G. M., \& White, H. (2003). Flexible Tool for Model Building: The Relevant Transformation of the Inputs Network Approach (RETINA). Oxford Bulletin of Economics and Statistics. https://ideas.repec.org/p/ucm/doicae/0309.html

Pérez-Amaral, T., Valarezo, Á., López, R., Garín-Muñoz, T., \& Herguera, I. (2020). Ecommerce by individuals in Spain using panel data 2008-2016. Telecommunications Policy, 44(4), 101888. https://doi.org/10.1016/j.telpol.2019.101888

Ruiz-Rodríguez, F., Lucendo-Monedero, A. L., \& González-Relaño, R. (2018). Measurement and characterization of the Digital Divide of Spanish regions at enterprise level. A comparative analysis with the European context. Telecommunications Policy, 42(3), 187-211.

https://doi.org/10.1016/j.telpol.2017.11.007

Salemink, K., Strijker, D., \& Bosworth, G. (2017). Rural development in the digital age: A systematic literature review on unequal ICT availability, adoption, and use in rural areas. Journal of Rural Studies, 54, 360-371. https://doi.org/10.1016/j.jrurstud.2015.09.001

Srinuan, C. \& Bohlin, E. (2011). Understanding the digital divide: A literature survey and ways forward. 22nd European Regional Conference of the International Telecommunications Society (ITS): Budapest, Hungary.

Szeles, M. R. (2018). New insights from a multilevel approach to the regional digital divide in the European Union. Telecommunications Policy, 42(6), 452-463.

https://doi.org/10.1016/j.telpol.2018.03.007

Valarezo, Á., Pérez-Amaral, T., Garín-Muñoz, T., Herguera García, I., \& López, R. (2018). Drivers and barriers to cross-border e-commerce: Evidence from Spanish individual behavior.

Telecommunications Policy, 42(6), 464-473. https://doi.org/10.1016/j.telpol.2018.03.006

Valarezo, Á., López, R., \& Pérez Amaral, T. (2020). Adoption of E-Commerce by Individuals and Digital-Divide. In J. Alleman, P. N. Rappoport, \& M. Hamoudia (Eds.), Applied Economics in the Digital Era: Essays in Honor of Gary Madden (pp. 103-134). Springer International Publishing. 
https://doi.org/10.1007/978-3-030-40601-1_4

van Dijk, J. A. G. M. (2006). Digital divide research, achievements and shortcomings. Poetics, 34(4), 221-235. https://doi.org/10.1016/j.poetic.2006.05.004

Vehovar, V., Sicherl, P., Hüsing, T., \& Dolnicar, V. (2006). Methodological Challenges of Digital Divide Measurements. The Information Society, 22(5), 279-290.

https://doi.org/10.1080/01972240600904076

Zhao, F., Collier, A., \& Deng, H. (2014). A multidimensional and integrative approach to study global digital divide and e-government development. Information Technology \& People, 27(1), 38-62. https://doi.org/10.1108/ITP-01-2013-0022

Zoroja, J. (2011). Internet, E-commerce and E-government: Measuring the Gap between European Developed and Post-Communist Countries. Interdisciplinary Description of Complex Systems, 9(2), 119133. 